Erik Esche, David Müller, Günter Wozny

\title{
Systematic modeling for optimization
}

Chapter in book | Accepted manuscript (Postprint)

This version is available at https://doi.org/10.14279/depositonce-11373

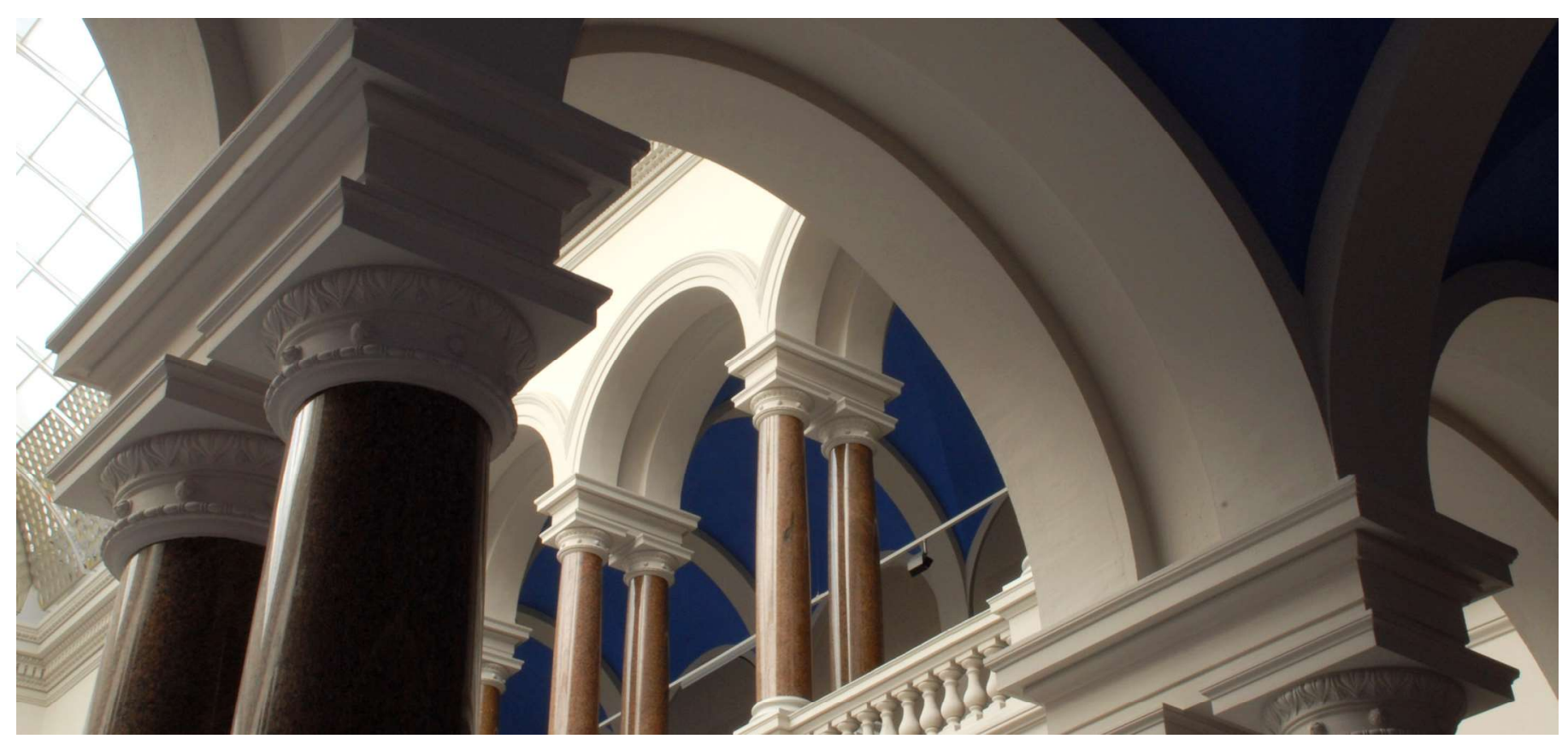

Esche, E., Müller, D. \& Wozny, G. (2014). Systematic Modeling for Optimization. Proceedings of the 8th International Conference on Foundations of Computer-Aided Process Design, 699-704.

https://doi.org/10.1016/b978-0-444-63433-7.50101-2 


\title{
Systematic Modeling for Optimization
}

\author{
Erik Esche* and David Müller, Günter Wozny \\ Chair of Process Dynamics and Operation, Technische Universität Berlin, \\ Straße des 17. Juni 135, Sekr. KWT-9, Berlin, 10623, Germany \\ *erik.esche@tu-berlin.de
}

\begin{abstract}
Optimization usually requires models, which are computationally speaking less expensive than models commonly used for simulations. At the same time, process optimization and model predictive control etc. require dependable accuracies in addition to the fastness. To demystify the art of preparing process models for optimization, a workflow is presented in this contribution, which systematically deduces models based on simplification of existing models and experiment based deduction of computationally inexpensive correlations.
\end{abstract}

Keywords: Multiple-Scale Modeling, Optimization, Convexification, Linearization

\section{Introduction}

Process systems engineers working in the field of optimization often find themselves in one of the following situations when modeling phenomena or an entire process: a large complex model exists, a short-cut model exists, or no model exists at all. Whatever the status quo is, the aim in the end is to have a model that not only describes the phenomena or process correctly, but has adequate numerical characteristics. Among these desired characteristics are a high degree of convexity and linearity. Often, process systems engineers will start randomly at various points of the project to develop or modify their model, i.e. perform experiments, establish an overly complex model not suitable for optimization, or forget to see the whole bigger picture by focusing on the phenomena level. A workflow systematizing the work of process engineers working on optimization models is required to firstly speed up the model development and model preparation process, to reduce redundancy or repetitiveness, and to aid in the general thinking process. Thus, a step towards systematizing the "art" of solving large-scale nonlinear programming problems is undertaken.

In this contribution, such a systematic workflow for the development of models for optimization purposes presented in [1] is expanded, whereby the workflow focuses on continuously differentiable models. Then, the workflow is applied on an absorption-desorption process. For this purpose, multi-stage model preparation is performed starting at the phenomena level and ending on the process level.

\section{Existing Strategies for Model Reduction}

Most solvers applied today for solving non-linear programming problems are gradient based methods and converge at fulfilled KKT conditions [2]. These conditions usually require the implementation of continuously differentiable models. This requirement is fulfilled by most classical short-cut models such as 
McCabe-Thiele method (McCabe \& Thiele, 1925). Their computation is also usually quite low. Only their accuracy leaves something to be desired. This aspect, on the other hand, is usually not an issue for models based on neural networks or support vector regression (Nandi et al.). In general, these types of models show a high accuracy for the operation points they were trained with. However, differentiability, interpolation, and especially extrapolation can be an issue here. Lastly, reduced order models (ROM) have become fashionable in recent years. Especially notable are Lang et al. (2009), who deduced ROMs from CFD simulations. The only disadvantages here are the expensive set-up procedure and the small area of applicability.

\section{Model Derivation for Optimization Purposes}

The model derivation for optimization is divided into two parts. Firstly, the general workflow for process systems engineers is presented and discussed. This is followed by a selection of possible strategies for improving the numerical behavior of systems.

\subsection{Parameter Identification and Subset Selection}

The workflow consists of 16 steps, which essentially should lead to a model suitable for optimization purposes. This whole workflow is described in [1] and shall just shortly be revisited here. The general idea is to firstly start off with the goal definition. The engineer must decide what type of model is required. This leads the engineer to Step 1. Here, the following question is stated: does a model for the system under investigation exist? If the answer is yes, this leads the engineer to the second question concerning the accuracy of the model (Step 2). If the accuracy is high enough, the model must be analyzed regarding its numerical convergence behavior. If this is also acceptable, then the model is already ready for optimization purposes. More details on all of the steps will be given in the following case study. 


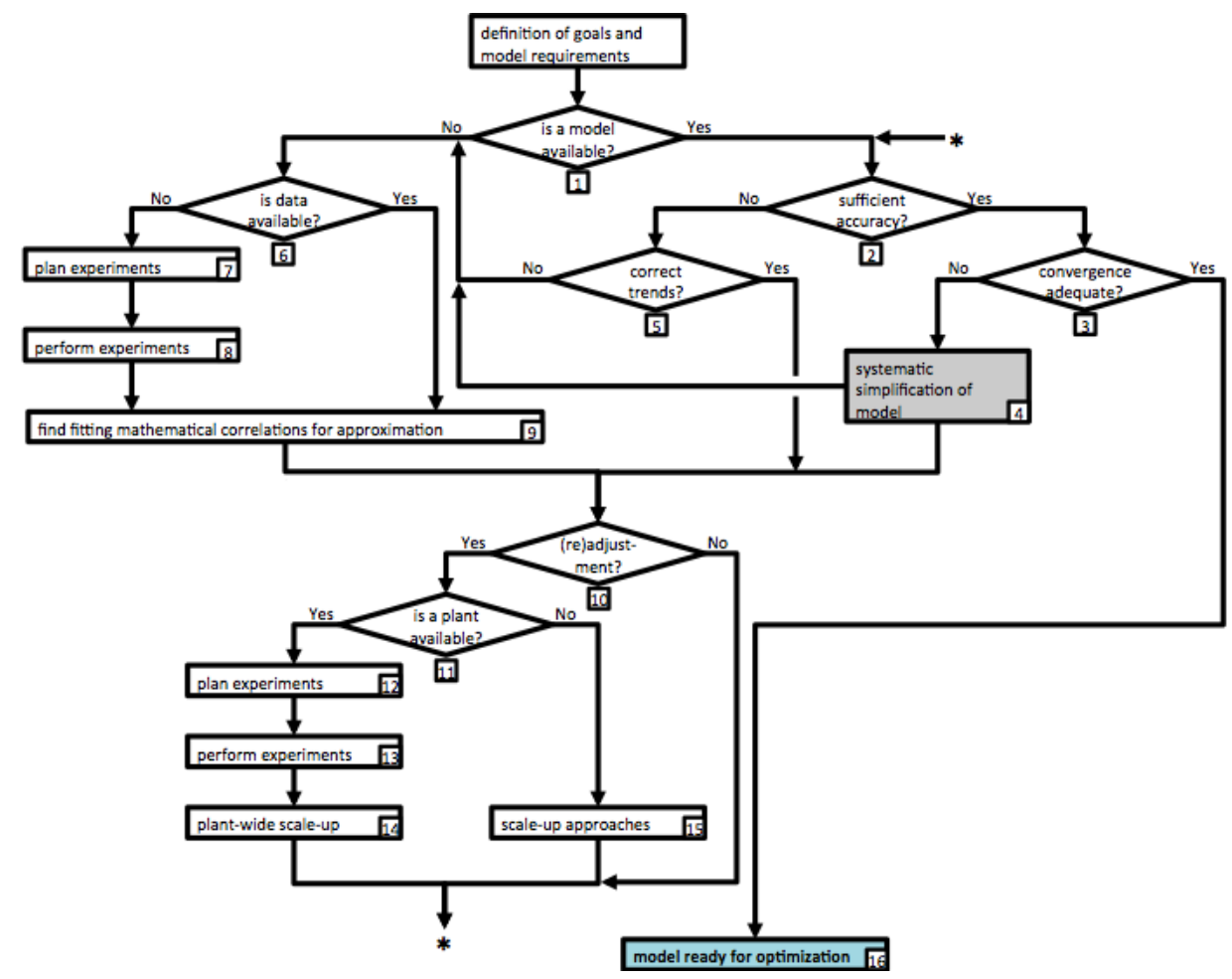

Figure 1. Systematic workflow for the development of models for optimization purposes [1].

\section{Case Study: Optimization Model of a $\mathrm{CO}_{2}$ Absorption Desorption Process}

The workflow shown above will now be applied to an absorption desorption process for the separation of $\mathrm{CO}_{2}$ from a product gas stream, which contains larger amounts of $\mathrm{C}_{2} \mathrm{H}_{4}$. The process model required is to be readied for superstructure optimization under uncertainty. In this context, a convergence time of below $1 \mathrm{sec}$ is demanded and a level of accuracy compared to experimental data of $\pm 5 \%$. The absorption of carbon dioxide $\left(\mathrm{CO}_{2}\right)$ using monoethanolamine (MEA) solutions is of course one of the more extensively investigated processes. The solubility of $\mathrm{CO}_{2}$ in aqueous solutions of MEA has been measured for a wide range of operating conditions as published by e.g. (Shen et al., 1992) and (Jou et al., 1995). The development of the respective kinetics started with (Clarke, 1964) and (Hikita et al., 1977). Simulation studies have been carried out with a high degree of complexity. Yeh et al. (1999) and Freguia et al. (2003) modeled the absorption rigorously and fitted their model to lab-scale and field data.

\subsection{Existing Mini-Plant}

At Technische Universität Berlin a mini-plant exists, which features a full-scale absorption desorption process for the removal of $\mathrm{CO}_{2}$. The absorption column has a packing height of $5 \mathrm{~m}$ and is operated at pressures of up to $30 \mathrm{bar}$. The desorption column has $4 \mathrm{~m}$ of packing and is operated at temperatures of up to $130^{\circ} \mathrm{C}$ at a pressure of up to $2.5 \mathrm{bar}$. Feed streams consisting of $\mathrm{CO}_{2}, \mathrm{CH}_{4}$, 
$\mathrm{C}_{2} \mathrm{H}_{4}$, and $\mathrm{N}_{2}$ have been stripped of $\mathrm{CO}_{2}$ at varying operating conditions using a $30 \mathrm{wt} \%$ monoethanolamine solution. The specific heat required for the removal of $\mathrm{CO}_{2}$ and the ethylene loss in the desorption section were recorded (Stünkel, 2013).

\subsection{Systematic Model Derivation}

The starting point of the algorithm shown in Fig. 1 has already been discussed. Also, given the extensive history of modeling MEA-based absorption of $\mathrm{CO}_{2}$, a rigorous model can easily be derived (Step 1). As part of this contribution ratebased simulations were implemented in Aspen Plus ${ }^{\circledR}$ using the ElectrolyteNRTL package in combination with correlations for the mass and heat transfer of the used package material (Mellapak 350 and 500) (Stünkel, 2013). The simulations incorporating all nine ionic and non-ionic liquid as well as the five gas components consist of roughly 2,000 state variables. To simulate a single steady-state operation point of the mini-plant, simulations of the absorption and desorption columns have to be prepared separately, before the recycles between each are slowly closed. In order to switch to a different operation point, the same procedure has to be repeated manually. Obviously, the convergence behavior of the rigorous model as implemented in Aspen Plus ${ }^{\circledR}$ is highly undesirable and not as required above. In addition, offsets greater than 10\% between model and experimental data from the mini-plant is observed, which answers the question in Step 2 about the sufficiency of the accuracy. The general behavior of the mini-plant can of course be expected to be seen in the rigorous model (Step 5). Hence, it is initially tried to simply readjust the existing model in Aspen Plus ${ }^{\circledR}$ (Step 10) to more closely mimic the experiments performed in the mini-plant (Steps 11 through 14). The most important step herein is the introduction of heat loss for each column. After this step, the accuracy of the Aspen Plus ${ }^{\circledR}$ model is satisfactory (Step 2). However, the convergence does not improve of course (Step 3).

\subsection{Systematic Simplification of the Model (Step 4)}

As described in (Esche et al., 2013) this step consists of a decomposition of an existing model and a systematic investigation of simplification potential in each different model part to ease the computational expense of the overall system. The main subdivision is the classification of the governing equations into mass balances, equilibrium equations, summations, energy and momentum balances, and auxiliary equations. In this case, the main source for the bad convergence behavior can be found in the bad scaling of the component balances. Several components such as $\mathrm{H}_{3} \mathrm{O}^{+}, \mathrm{OH}^{-}$, and $\mathrm{HCO}_{3}{ }^{2+}$ appear in tiny, but varying amounts, close to zero, i.e. $10^{-9}$ to $10^{-4} \mathrm{~mol} / \mathrm{m}^{3}$. Attempts to ease these troubles by manually scaling the respective equations proved to be in vain as the concentrations may vary depending on the state of the chemical equilibrium at different positions in the plant. As these troubles cannot be resolved so that the model does not repeatedly run into local infeasibilities, the decision was made to find a formulation of the entire system which does without the trace components. Given their necessity for the entire kinetic system a completely new description thereof is required. Within Step 4 this leads to the decision to acquire experimental data for the phenomena level.

4.4. Modeling at the Phenomena Level

Given the extensive level MEA-based $\mathrm{CO}_{2}$ absorption has already been investigated, experimental data is available to great extents (Step 6). Therefore, 
planning and performing lab-scale experiments can actually be skipped at this point (Steps 7 and 8). Data published by Shen et al. (1992) and Kim (2007) on the solubility of $\mathrm{CO}_{2}$ in $30 \mathrm{wt} \%$ MEA solutions and the heat of absorption respectively is employed to derive bivariate correlations. Fig. 2 shows the experimental data (blue lines) and the respective correlations (colored surfaces) for each (Step 9).
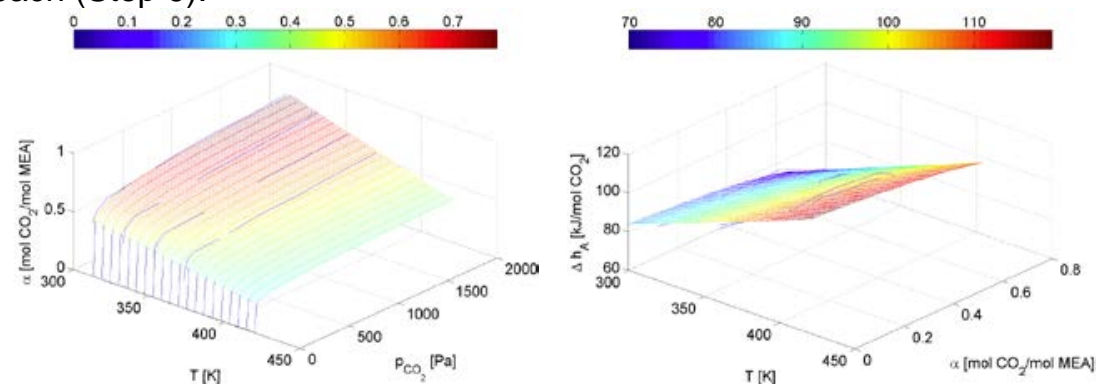

Figure 2. Solubility of $\mathrm{CO}_{2}$ (left) in a 30 wt.-1\% MEA solution $\alpha$ depending on temperature $\mathrm{T}$ and the partial pressure of $\mathrm{CO}_{2} \mathrm{p}_{\mathrm{CO} 2}$ and heat of absorption of $\mathrm{CO}_{2} \Delta \mathrm{h}_{\mathrm{A}}$ (right) depending on temperature $T$ and the solubility $\alpha$.

The underlying functions of the correlations are determined with respect to the general curvature of the data. For this purpose the bivariate dependency is especially useful. Based on the solubility and heat of absorption correlations no description of the ionic composition is required. The system is reduced to three liquid components $\left(\mathrm{CO}_{2}, \mathrm{MEA}\right.$, and $\left.\mathrm{H}_{2} \mathrm{O}\right)$ and four gas components $\left(\mathrm{CO}_{2}, \mathrm{H}_{2} \mathrm{O}\right.$, $\mathrm{CH}_{4}$, and $\mathrm{C}_{2} \mathrm{H}_{4}$ ). At the phenomena level these correlations are joined with a basic description of the vapor pressure of water using the Antoine equation, a correlation estimating the solubility of $\mathrm{C}_{2} \mathrm{H}_{4}$ in MEA solutions derived from data published by Carroll et al. (1998). At this point, no additional information will be given on the actual modeling seeing as it has already been published in (Esche et al., 2013).

\subsection{Fitting to Mini-plant Data}

To model the whole mini-plant for the absorption desorption process, the absorption column is divided into 40 equilibrium stages, for which component mole balances are formulated as well as energy balances. One equilibrium stage is used to model both the flash and the desorption column. Tray efficiencies are introduced into the solubility equations for each equilibrium stage. In addition, $U * A$-based heat loss correlations and the heat of evaporation of water are added. The liquid recycle from the desorption column back to the absorption is modeled as a forwarding of the $\mathrm{CO}_{2}$ load, whilst MEA and $\mathrm{H}_{2} \mathrm{O}$ are reinitiated to ease the computational complexity. This extended model is then fitted to actual experimental mini-plant data reported by Stünkel (2013). The experiments were carried out in the mini-plant varying the absorption pressure between 5 and 32 bar, the gas load factor between 0.25 and $0.42 \mathrm{~Pa}^{0.5}$, the feed concentrations of carbon dioxide between 0.14 and $0.26 \mathrm{~mol} / \mathrm{mol}$, the $\mathrm{CO}_{2}$ removal rate between 70 and $100 \%$, as well as the flow of the scrubbing liquid from 10 to $60 \mathrm{~kg} / \mathrm{h}$. It was found, that all parameters above can be set to constant values, while the efficiency for the desorption column can be described depending on five characteristic variables: the absorption pressure, the gas load factor, the feed concentration of $\mathrm{CO}_{2}$, the removal rate of $\mathrm{CO}_{2}$, and the 
scrubbing liquid flow. For the subsequent parameter estimation the solubility correlation parameters, the stage efficiencies, and $U * A$ are used as decision variables. This concludes Steps 10 to 14 in the systematic in Fig. 1.

\subsection{Numerical Stabilization Strategies}

The resulting, fitted model can represent all experimental points with a margin of error of $\pm 3 \%$ with respect to the energy required per captured kilogram of $\mathrm{CO}_{2}$. So the reevaluation in Step 2 gives a positive reply. However, when given to Step 3, the model at this point still is not adequate. For this reason, it is handed back to Step 4, in which at this point no simplifications but rather reformulations are carried out. Among these are:

$$
\begin{aligned}
& \frac{a}{b} \Rightarrow \frac{a}{b+\varepsilon}, \varepsilon>0 \\
& \frac{a}{b} \Rightarrow \frac{a}{\max (0, b)}, \max (0, b) \approx \frac{1}{2} \cdot b+\frac{1}{2} \cdot \sqrt{b^{2}+\varepsilon}, \varepsilon>0 \\
& a^{b} \Rightarrow(\max (0, a))^{b}, \max (0, a)=\frac{1}{2} \cdot a+\frac{1}{2} \cdot \sqrt{a^{2}+\varepsilon}, \varepsilon>0 \\
& \log _{a}(b)=\ln (b) / \ln (a)=c \Rightarrow b=\exp (c \cdot \ln (a))
\end{aligned}
$$

Eq. (1) and (2) are applied as reformulations for various fractions appearing in the system. Whenever $b$ can be expected to take values close to 0 , the reformulation from Eq. (2) is employed. This substitution is more computationally expensive but stabilizes the fraction more reliably by also fixing the non-differentiability. Eq. (1) instead does not rectify this, but seems to be sufficient for most cases, in which $b$ only infrequently turns zero during an optimization or simulation iteration step. The substitution in Eq. (3) similarly is only applied to cases, in which a is frequently smaller than zero. Lastly, Eq. (4) is employed for reformulating equations which can be written explicitly in terms of the logarithmic function. For the model derived here these four reformulations were chosen. It would also have been possible to substitute the problematic terms in the equations and add additional equations for those. However, seeing as the system in hand is already quite sparse, the additional equations would only increase the computational complexity as there would be no additional benefit from a further increase in sparsity. Optimization studies carried out with this model showed a fast and reliable convergence from different starting points and usually converged within a second on a 64bit AMD Athlon X2 Dual Core Processor $3800+$. Hence, with respect both to accuracy and computational time (Steps 2 and 3), this model is suitable for optimization.

\section{Conclusions and Outlook}

So far the systematic introduced in this article has been applied to two fundamentally different systems and applied successfully to derive computationally fast and accurate models for optimization. For future work, the workflow will be reapplied to more complex systems and extended if necessary. Additionally, the numerical stabilization strategies will be extended and formalized further. The workflow as a whole will be implemented into the online modeling platform MOSAIC. 


\section{Acknowledgments}

The authors acknowledge the support from the Cluster of Excellence "Unifying Concepts in Catalysis" and the Collaborative Research Center SFB/TR 63 InPROMPT "Integrated Chemical Processes in Liquid Multiphase Systems" both coordinated by the Technische Universität Berlin and funded by the German Research Foundation (Deutsche Forschungsgemeinschaft "DFG").

\section{References}

J.K.A Clarke, 1964, Kinetics of absorption of carbon dioxide in monoethanolamine solutions at short contact times, Ind. Eng. Chem. Fundamen., 3, $239-245$

J.J. Carroll, J. Maddocks, A.E. Mather, 1998, The Solubility of Hydrocarbons in Amine Solutions, Laurance Reid Gas Conditioning Conference, Norman, Oklahoma

E. Esche and D. Müller, R. Kraus, G. Wozny, 2013, Systematic Approaches for Model Derivation for Optimization Purposes, Chemical Engineering Science. DOI: 10.1016/j.ces.2013.11.041.

S. Freguia, G.T. Rochelle, 2003, Modeling of CO2 capture by aqueous monoethanolamine, AIChE Journal, 49, 1676 - 1686

H. Hikita, S. Asai, H. Ishikawa, M. Honda, 1977, The kinetics of reactions of carbon dioxide with monoethanolamine, diethanolamine and triethanoamine by a rapid mixing method, The Chemical Engineering Journal, 13, 7 - 12

F.-Y. Jou, A.E. Mather, F.D. Otto, 1995, The solubility of CO2 in a 30 mass percent monoethanolamine solution, The Canadian Journal of Chemical Engineering, 73, 140 $-147$

I. Kim, H.F. Svendsen, 2007, Heat of absorption of carbon dioxide (CO2) in monoethanolamine (MEA) and 2-(aminoethyl)ethanolamine (AEEA) solutions, Ind. Eng. Chem., 46, $5803-5809$

H. W. Kuhn, A. W. Tucker, Nonlinear programming, 1951, Proceedings of the $2^{\text {nd }}$ Berkeley Symposium, University of California Press, 481 - 492.

S. Kuntsche, H. Arellano-Garcia, G. Wozny, 2011,MOSAIC, an environment for webbased modeling in the documentation level, Computer Aided Chemical Engineering 29, 1140-1144.

Y. Lang, A. Malcina, L.T. Biegler, S. Munteanu, J.I. Madsen, S.E. Zitney, 2009, Reduced order model based on principal component analysis for process simulation and optimization. Energy \& Fuels, 23, 1695 - 1706

S. Nandi, Y. Badhe, J. Lonari, U. Sridevi, B. Rao, S.S. Tambe, B.D. Kulkarni, 2004, Hybrid process modeling and optimization strategies integrating neural networks/support vector regression and genetic algorithms: study of benzene isopropylation on hbeta catalyst, Chem. Eng. Trans., 29, 1495 - 1500

W. McCabe, E. Thiele, 1925, Graphical design of frationating columns, Industrial \& Engineering Chemistry, 20, $591-609$

K.P. Shen, M.H. Li, 1992, Solubility of carbon dioxide in aqueous mixtures of monoethanolamine with methyldiethanolamine, J. Chem. Eng. Data, 37, 96 - 100

S. Stünkel, 2013, Kohlendioxid-Abtrennung in der Gasaufbereitung des Prozesses der oxidativen Kupplung von Methan, PhD Thesis, Technische Universtität Berlin

A.C. Yeh, H. Bai, 1999, Comparison of ammonia and monoethanolamine solvents to reduce $\mathrm{CO} 2$ grenhouse gas emissions, Science of the Total Environment, 228, 121 131 